\title{
Amelioration De La Maturite Des Entreprises En Gestion Des Risques Par La Maintenance
}

\author{
Hakim Nissoul, MSc \\ Laboratoire Mécanique, Management Industriel et Innovation, \\ Faculté des Sciences et Techniques de Settat, \\ Université Hassan Premier, Settat, Maroc \\ Fouad Riane, PhD \\ Laboratoire Mécanique, Management Industriel et Innovation, \\ Faculté des Sciences et Techniques de Settat, \\ Université Hassan Premier, Settat, Maroc \\ Laboratoire de Génie Industriel, Centrale Supéléc \\ Ecole Centrale Casablanca, Route de Nouasseur, \\ Sidi Maârouf, Casablanca, Maroc \\ Ahmed Mousrij, PhD \\ Laboratoire Mécanique, Management Industriel et Innovation, \\ Faculté des Sciences et Techniques de Settat, \\ Université Hassan Premier, Settat, Maroc
}

Doi: 10.19044/esj.2017.v13n30p33 URL:http://dx.doi.org/10.19044/esj.2017.v13n30p33

\begin{abstract}
Statistics on accidents at work, particularly in Morocco, remain worryingly high. This situation raised a general awareness in the country and many decisions have been taken and corrective actions have been elaborated in order to enhance enterprise risk management maturity. This maturity is based on several key factors including maintenance.In this paper we propose a methodology to mitigate and control maintenance generated risks which in turn will allow enterprises to enhance their maturity in risk management.
\end{abstract}

Keywords: Industrial risk, maintenance, maturity, risk treatment

\section{Résumé}

Les statistiques sur les accidents liés au travail, particulièrement au Maroc, demeurent inquiétantes. Cette situation a induit une prise de conscience générale dans le pays et plusieurs actions ont été initiées pour améliorer le niveau de maturité des entreprises en gestion des risques industriels, cette maturité est basée sur plusieurs facteurs clés dont notamment la maintenance. Nous proposons dans le présent papier une 
méthodologie permettant de maitriser les risques liés à la maintenance ce qui permettra d'aider les entreprises à faire évoluer leurs niveau de maturité en gestion des risques industriels.

Mots-clés : Risques industriels; maintenance; maturité; traitement des risques

\section{Introduction}

Les statistiques sur les accidents liés au travail sont inquiétantes. Selon l'Organisation Internationale de Travail (OIT), environ 2,3 millions de décès par an dans le monde à cause d'un accident ou d'une maladie lié au travail (OIT, 2015). Le coût humain de cette menace quotidienne est considérable et on estime que le fardeau économique des mauvaises pratiques de sécurité et santé au travail représente, tous les ans, (4\%) pour cent du produit intérieur brut (PIB) mondial soit le montant astronomique de 2800 milliards de dollars (Pour mémo PIB mondial en 2016 : 75.000 milliards de dollars) (OIT, 2017). Ce coût couvre le temps de travail perdu, les interruptions de production et les frais de traitement, de réadaptation et d'indemnisation occasionnés par les lésions et maladies professionnelles (OIT, 2015).

Les décès et les blessures frappent très lourdement les pays en développement, où une grande partie de la population exercent des activités dangereuses (OIT, 2013). Les chiffres liés aux accidents de travail sont encore plus inquiétants quand ils sont rapportés au contexte marocain. Le Maroc est classé par l'organisation internationale du travail comme un des pays les plus dangereux de la région MENA (Middle East and North Africa) pour les travailleurs (Elkholti, 2015). Cette situation inquiétante a induit une prise de conscience générale dans le Maroc et plusieurs actions ont été initiées en matière de prévention, d'appréciation et de contrôle de la sécurité des sites industriels et commerciaux visant à améliorer la maturité des entreprises en gestion des risques industriels (Madika, 2011).

Cette maturité est basée sur plusieurs facteurs clés dont notamment la maintenance (Nissoul et al., 2017). En effet, Entre 10 et 15\% des accidents professionnels mortels et entre 15 et $20 \%$ de la totalité des accidents seraient liés aux travaux de maintenance (FAT, 2010). Contrairement aux activités de production, les activités de maintenance nécessitent toujours des interactions directes avec les équipements, les biens ou processus à maintenir. Les personnels de maintenance doivent se rendre sur place, à proximité de ces équipements ou processus. Ils n'ont donc pas de poste de travail unique et fixe et sont très mobiles. Ils sont ainsi exposés aux risques associés à cette forte mobilité, aux risques présentés par les équipements ou systèmes, et par les ambiances dans lesquelles ces équipements se trouvent (INRS, 
2016).Comme le soulignent Ray et al., les efforts pour prévenir les risques industriels se sont historiquement focalisés sur les opérations de production (Ray et al., 2000). Aujourd'hui, avec l'automatisation accrue, la complexité et le coût croissants des équipements, et par conséquent la nécessité de réduire les temps d'indisponibilité de ces derniers, la maintenance prend une importance croissante, et il convient de développer la prévention dans ce domaine (INRS, 2005). Dans cette perspective, nous proposons une méthodologie permettant de maitriser les risques liés à la maintenance, avec un exemple d'application concret.

L'objet du paragraphe qui suit est de présenter la maintenance en rappelant sa définition, ses différentes formes ainsi qu'un aperçu général sur l'évolution des approches de maintenance avant de présenter la méthodologie que nous proposons pour la maitrise des risques liés à la maintenance.

\section{Gestion de la maintenance}

Le fascicule de documentation «FD X 60-000: Maintenance Industrielle »d'Afnor définit la maintenance comme étant l'ensemble de toutes les actions techniques, administratives et de management durant le cycle de vie d'un bien, destinées à le maintenir ou à le rétablir dans un état dans lequel il peut accomplir la fonction requise (Afnor, 2002).

Le management de la maintenance concerne toutes les activités des instances de direction qui d'une part déterminent les objectifs, la stratégie et les responsabilités concernant la maintenance, et d'autre part les mettent en application par des moyens tels que la planification, la maîtrise et l'amélioration des méthodes de maintenance dans l'entreprise (Glade, 2005).

Selon l'INRS, la maintenance se présente sous plusieurs formes, on distingue la maintenance préventive, la maintenance correctives et la maintenance à échelle majeure (cf. Figure 1) (INRS, 2013).

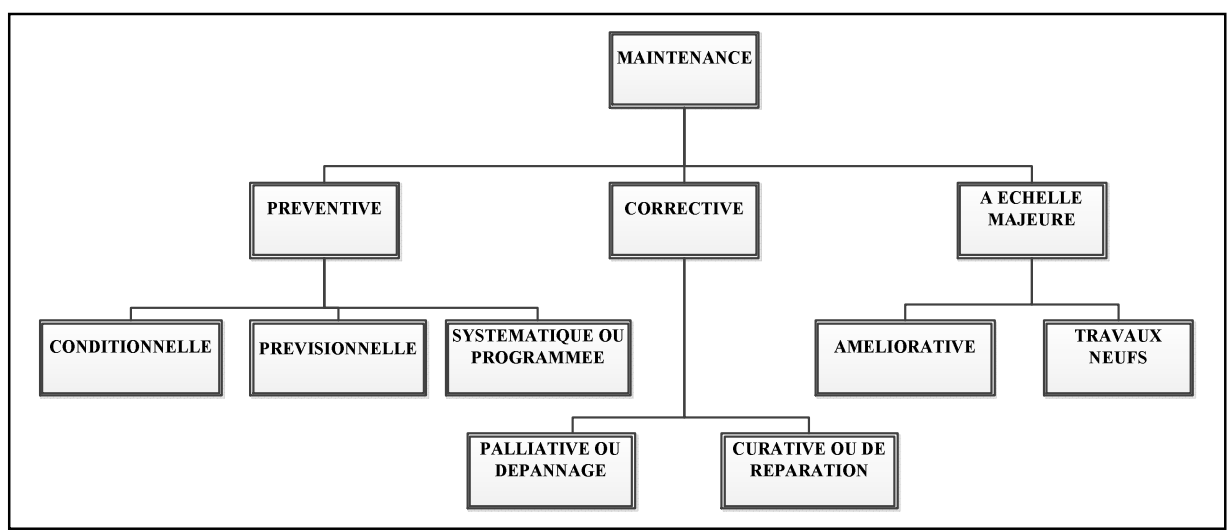

Figure 1 : Les différentes formes de la maintenance (adapté de (INRS, 2013)) 
La maintenance préventive est exécutée à des intervalles prédéterminés ou selon des critères prescrits et destinés à réduire la probabilité de défaillance ou la dégradation du fonctionnement d'un bien, elle est subdivisée en la Maintenance conditionnelle qui est basée sur une surveillance du fonctionnement du bien et/ou des paramètres significatifs de ce fonctionnement et intégrant les actions qui en découlent, la Maintenance prévisionnelle qui est exécutée en suivant les prévisions extrapolées de l'analyse et de l'évaluation de paramètres significatifs de la dégradation du bien et en la Maintenance systématique qui est exécutée à des intervalles de temps préétablis ou selon un nombre défini d'unités d'usage mais sans contrôle préalable de l'état du bien (Afnor, 2002).

La Maintenance corrective est exécutée après détection d'une panne et destinée à remettre un bien dans un état dans lequel il peut accomplir une fonction requise (Afnor, 2002). Elle est subdivisée en la Maintenance palliative ou dépannage qui est destinée à permettre à un bien d'accomplir provisoirement tout ou partie d'une fonction requise et en la Maintenance curative ou de réparation qui a pour objet de rétablir un bien dans un état spécifié pour lui permettre d'accomplir une fonction requise, le résultat des actions réalisées doit présenter un caractère permanent (INRS, 2013).

Dans le milieu industriel, la combinaison entre maintenance préventive et maintenance corrective est à la fois nécessaire et implicite. En effet, la maintenance préventive peut réduire la probabilité de défaillance mais ne pourra pas juguler totalement la défaillance qui arrive aléatoirement. L'augmentation du nombre d'intervention préventive, (cf. Figure 2), permet de réduire les coûts et impacts de l'indisponibilité non maitrisée du système mais provoque une augmentation du coût global par le biais de coûts d'inspection et d'exécution des maintenances préventives. Un des challenges pour le management est de trouver la balance optimale (Nguyen, 2012).

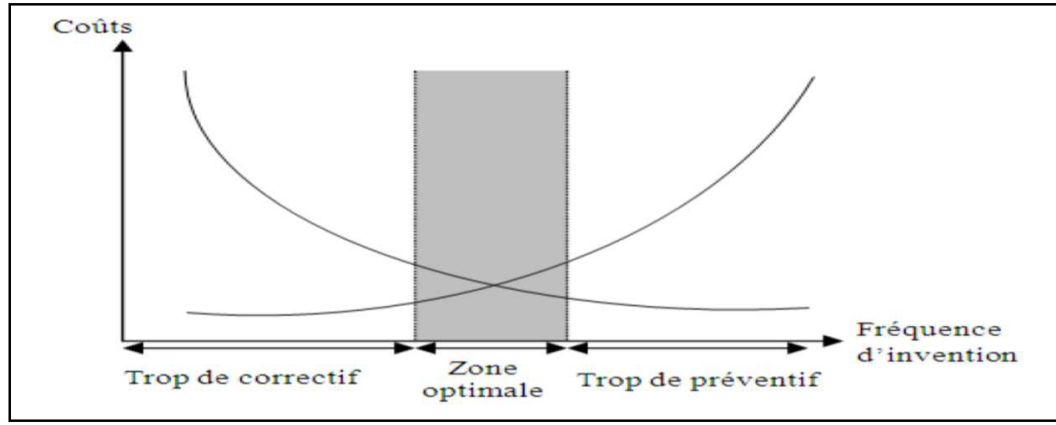

Figure 2 : La balance entre maintenance corrective et préventive (Nguyen, 2012) 
La maintenance à échelle majeure est effectuée dans le but de permettre l'accomplissement par le bien de fonctions nouvelles ou supplémentaires, ou des mêmes fonctions dans de meilleures conditions. Elle est subdivisée en la Maintenance améliorative qui vise à améliorer la fiabilité et la maintenabilité du système ou à modifier un équipement ou un sous ensemble de façon à augmenter sa disponibilité et en les travaux neufs qui sont des travaux de construction, d'installation, de mise au point et de mise en service après installation de nouveaux biens ou équipements (INRS, 2013).

Face au développement technologique et à la complexité accrue des processus de production dans un environnement de plus en plus concurrentiel, les approches de maintenance ont évolué. De nombreux travaux de synthèse présentent les différentes politiques de maintenance et leur applicabilité en contexte opérationnel (Nguyen, 2012; Monchy \& Vernier, 2010).Ces approches sont classées en quatre générations, (Arunraj, \& Maiti, 2007), à savoir :

La première génération concerne la période avant les années 1950, pendant laquelle l'industrie a commencé ses premiers pas de développement. La mécanisation était très limitée et les systèmes étaient généralement de conception simple et surtout très spécifiques. Les stratégies de maintenance consistaient essentiellement à faire fonctionner les systèmes jusqu'à la défaillance pour ensuite les réparer ou éventuellement les remplacer (Maintenance corrective).

La deuxième génération concerne la période entre les années 1950 et 1980, ladite période est caractérisée par l'évolution de la technologie en nombre et en complexité pour satisfaire à la demande croissante de biens, d'énergie et de transports. Sur le plan de la maintenance, ceci se traduit aussi par une meilleure maitrise des systèmes de production et la définition des premières politiques de maintenance préventive.

La troisième génération concerne la période entre les années 1980 et 2000, elle est caractérisée par la complexité accrue des processus de production, l'usage croissant des systèmes automatisés et des approches de juste à temps. Sur le plan de la maintenance, ceci se traduit par la priorisation des actions de maintenance et l'identification de manière quasi-systématique des causes des défaillances. C'est le principe de la maintenance basée sur la fiabilité qui est fondée sur la connaissance du système, la recherche des modes de défaillances et la prise d'actions proactives visant à éliminer les causes de défaillance.

La quatrième génération concerne la période après les années 2000, elle est caractérisée par la prise en considération des contraintes sécurité.

L'objectif est d'assurer l'exploitation maximale des systèmes de production tout en respectant les exigences sécurité. C'est le principe de la 
maintenance basée sur les risques qui sera détaillé dans le paragraphe suivant.

\section{Maintenance basee sur les risques}

La méthode de la maintenance basée sur les risques a pour but de réduire les risques des systèmes de production par la maintenance et elle a été appliquée initialement dans les domaines pétroliers et gaziers (cf. Figure 3) (Khan \& Haddara, 2004). Cette méthode est composée des principales étapes suivantes (Arunraj \& Maiti, 2007) :

Etape 1- Division du système en unité gérable: Cette étape consiste en la décomposition du système de production en unité opérationnelle facilement gérable.

Etape 2- Sélection d'une unité : Cette étape consiste en la sélection d'une unité opérationnelle à partir des unités définies lors de l'étape précédente.

Etape 3 - Identification des risques : Cette étape consiste en la recherche, la reconnaissance et la description des risques (ISO, 2009), auxquelles est exposée l'unité opérationnelle sélectionnée.

Etape 4 - Estimation des conséquences : Pour chaque risque identifié, cette étape consiste en la description du dommage subi, et l'évaluation de la gravité de ce dommage.

Etape 5 - Estimation de la probabilité : pour chaque risque identifié, cette étape consiste en l'évaluation de la probabilité de survenance du risque en se basant sur le retour d'expériences.

Etape 6 - Evaluation du risque : pour chaque risque identifié, cette étape consiste en l'évaluation du niveau de risque qui est le produit de la conséquence et de la probabilité d'occurrence.

Etape 7 - Evaluation de l'acceptabilité du risque : cette étape consiste en la détermination des critères d'acceptabilité des risques et évaluation de l'acceptabilité sur la sécurité à l'aide de la matrice de criticité par exemple.

Etape 8 - Planning de maintenance : $\mathrm{Si}$ le risque est non acceptable, cette étape consiste en la définition de la modalité de maintenance permettant de réduire le risque à un niveau acceptable et l'intégrer dans le planning de maintenance.

Etape 9 - Sélection d'une autre unité : Si le risque est acceptable, cette étape consiste en la réalisation de l'étude pour une autre unité opérationnelle du système de production jusqu'à ce que toutes les unités identifiées soient étudiées. 


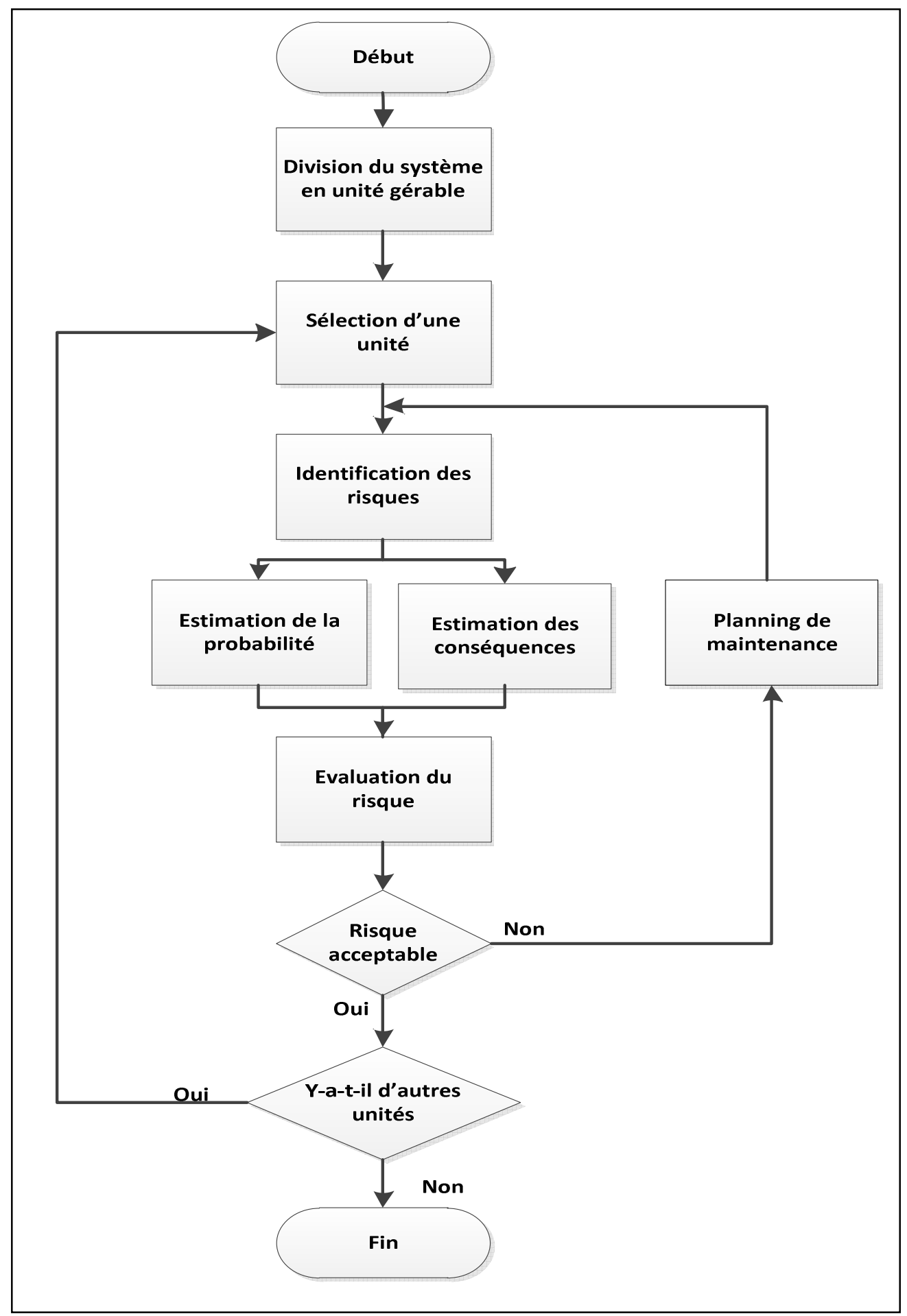

Figure 3 : Maintenance basée sur les risques (Traduit de (Arunraj \& Maiti, 2007)) 
Les approches existantes Dans la littérature (Nilsson, 2003; Sadiq et al., 2004; Vaurio, 1995; Jinqiu \& Zhang, 2014), utilisent le risque comme un critère de planification de la maintenance, d'optimisation des coûts et d'amélioration de la fiabilité des équipements et installations mais les risques induits par la maintenance ne sont pas pris en considération. Dans le paragraphe qui suit, nous allons détailler l'approche que nous proposons pour maitriser les risques liés à la maintenance.

\section{Approche proposee}

Nous proposons une approche, inspirée des travaux de Hadni concernant la méthodologie "MIS » (impact de la maintenance sur la sécurité). Cette méthodologie représente un nouveau concept qui intègre en plus d'une analyse classique du risque associé à l'exploitation ou au process, une analyse des risques induits par la maintenance, elle vise l'évaluation globale du risque et l'optimisation du choix de la périodicité de la maintenance (Hadni, 2015). Cependant, l'évaluation globale des risques n'est pas toujours nécessaire car les risques liées à la maintenance et ceux liées à l'exploitation peuvent être traités séparément. Aussi, l'optimisation de la périodicité de la maintenance vise à améliorer la fiabilité des équipements et réduire ainsi les risques associés à l'exploitation mais elle pourra faire augmenter les risques associés à la maintenance notamment en cas d'accroissement de la périodicité de la maintenance. Dans certains cas, il faudra changer complétement les mesures de maintenance afin de réduire le risque à un niveau acceptable.

L'approche que nous proposons permets de maitriser les risques liés à la maintenance d'une manière générale, y compris dans le cas de la méthode de la maintenance basée sur les risques où on vise à réduire les risques process par la maintenance. Lorsque les risques générés par les opérations de maintenance sont non acceptables, on procède à un changement total desdites opérations de maintenance ou à leurs améliorations par l'ajout par exemple des moyens de protection ou par la réalisation de formation, jusqu'à ce que les risques générés par lesdites opérations de maintenance deviennent acceptables. Par ailleurs, nous proposons d'assurer une veille continue qui permettra à la suite de tout changement d'environnement, ou de développement technologique, de mettre à jour les évaluations des risques et d'assurer en permanence une amélioration continue (cf. Figure 4). 


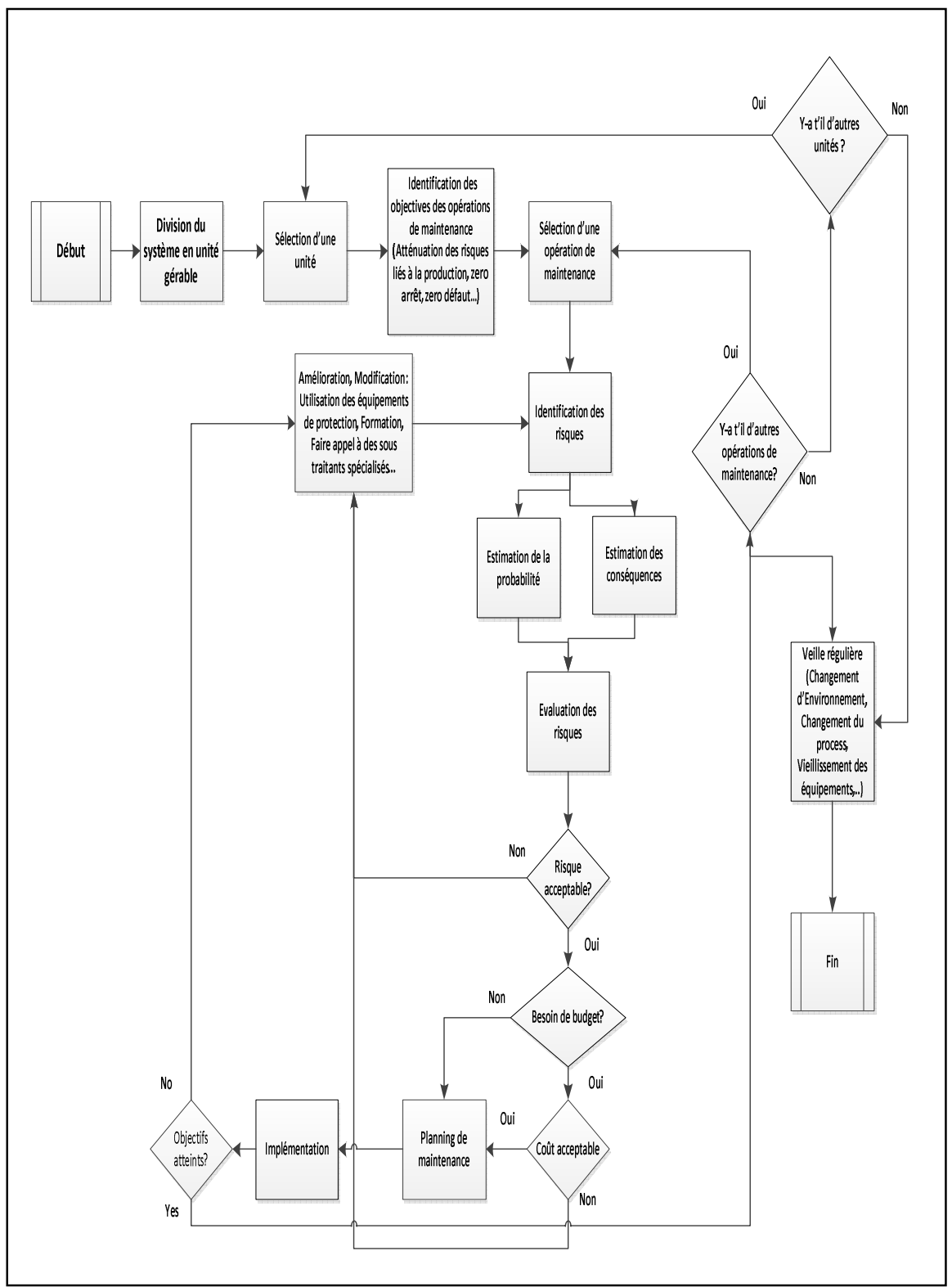

Figure 4 : Méthodologie de maitrise des risques liés à la maintenance

L'approche proposée est composée des étapes suivantes :

Etape 1- Division du système en unité gérable: Cette étape consiste en la décomposition du système de production en unité opérationnelle facilement gérable.

Etape 2- Sélection d'une unité : Cette étape consiste en la sélection d'une unité opérationnelle à partir des unités définies lors de l'étape précédente. 
Etape 3 - Identification des opérations de maintenance : Cette étape consiste en l'identification des opérations de maintenance relatives à l'unité sélectionnée qui permettront d'atteindre des objectifs prédéfinis (Par exemple : atténuation du risque lié à la production pour le cas de la méthode de la maintenance basée sur les risques, amélioration de la durée de vie de l'installation, amélioration de la disponibilité de l'installation...).

Etape 4 - Sélection d'une opération de maintenance : Cette étape consiste en la sélection d'une opération de maintenance à partir des opérations de maintenance définies lors de l'étape précédente.

Etape 5 - Identification des risques liés aux opérations de maintenance : Cette étape consiste en la recherche, la reconnaissance et la description des risques (ISO, 2009), liés à l'exécution de l'opération de maintenance sélectionnée.

Etape 6 - Estimation des conséquences : pour chaque risque identifié, cette étape consiste en la description du dommage subi, et évaluation de la gravité de ce dommage.

Etape 7 - Estimation de la probabilité : pour chaque risque identifié, cette étape consiste en l'évaluation de la probabilité de survenance du risque en se basant sur le retour d'expériences.

Etape 8 - Evaluation du risque : pour chaque risque identifié, cette étape consiste en l'évaluation du niveau de risque qui est le produit de la conséquence et de la probabilité d'occurrence.

Etape 9- Evaluation de l'acceptabilité du risque : Cette étape consiste en la détermination des critères d'acceptabilité des risques et l'évaluation de l'acceptabilité sur la sécurité à l'aide de la matrice de criticité.

Etape 10-Analyse de la rentabilité économique : Dans la majeure partie des cas, la décision en maintenance repose essentiellement sur une contrainte budgétaire (Deloux, 2008 ; Meddaoui, 2013).En effet, la sélection des meilleures opérations de maintenance n'est pas suffisante, il faut aussi savoir choisir les plus économiques (Barthelemy \& Courreges, 2012). Pour ceci, même si le risque lié à l'opération de maintenance est jugé acceptable, cette étape consiste en l'analyse et la vérification de l'acceptabilité du coût d'investissement par rapport à la criticité (fréquence $\mathrm{x}$ gravité) du risque.

Etape 11- Maintien ou intégration de l'opération de maintenance dans le planning de maintenance : Si le risque et le coût d'investissement sont acceptables, cette étape consiste en le maintien ou l'intégration de l'opération de maintenance dans le planning de maintenance tout en assurant une veille continue qui permettra à la suite de tout changement d'environnement, ou de développement technologique, de mettre à jour les évaluations des risques et d'assurer en permanence une amélioration continue. 
Etape 12- Sélection d'une autre opération de maintenance : Si le risque et le coût d'investissement sont acceptables, cette étape consiste en la réalisation de l'étude pour une autre opération de maintenance jusqu'à ce que toutes les opérations de maintenance identifiées soient étudiées.

Etape13- Modification/amélioration de l'opération de maintenance : Si le risque liée à l'exécution de l'opération de maintenance et/ou le coût d'investissement sont non acceptables, cette étape consiste au changement total de ladite opération de maintenance ou à son amélioration par l'ajout par exemple des moyens de protection ou par la réalisation de formation, jusqu'à ce que les risques générés par la dite opération de maintenance et/ou le coût d'investissement seront réduits à un niveau acceptable.

Etape 14 - Mise en ouvre du planning de maintenance : Une fois tous les risques et tous les coûts d'investissement liés à l'exécution de toutes les opérations de maintenance sont réduits à un niveau acceptable, cette étape consiste en la mise en œuvre desdites opérations de maintenance et on la réévaluation du niveau d'atteinte des objectifs escomptés pour s'assurer de leur efficacité.

Dans le paragraphe suivant, nous allons présenter un exemple d'application de la méthodologie proposée.

\section{Exemple d'application}

Après analyse de l'exemple étudiée par Hadni qui concerne l'unité de déchargement d'ammoniac situé au niveau d'un groupe Marocain leader dans le domaine de l'industrie chimique (Hadni, 2015). nous avons choisi d'appliquer notre méthodologie au bac de stockage d'ammoniac qui est, selon les résultats de l'étude de Hadni, l'équipement le plus critique pour lequel le risque n'a pas pu être réduit à un niveau acceptable malgré l'optimisation de la périodicité de la maintenance.

L'unité de déchargement est située à proximité de la ville et d'une route nationale fréquentée située au nord de la station. Du côté sud de cette dernière se situe l'unité de stockage du soufre en poudre. Alors que du côté ouest se trouve l'unité de production du soufre liquide. Une autre industrie est installée à l'est de la station d'ammoniac. Quatre pipe-lines qui assurent le transfert de certains produits traversent la station d'NH3.

L'application de l'approche proposée «Maitrise des risques liés à la maintenance » à l'exemple étudié est décrite ci-dessous.

Etape 1 - Décomposition du système en unités gérable : La figure 5 schématise la décomposition de l'unité de déchargement d'ammoniac en équipements. 


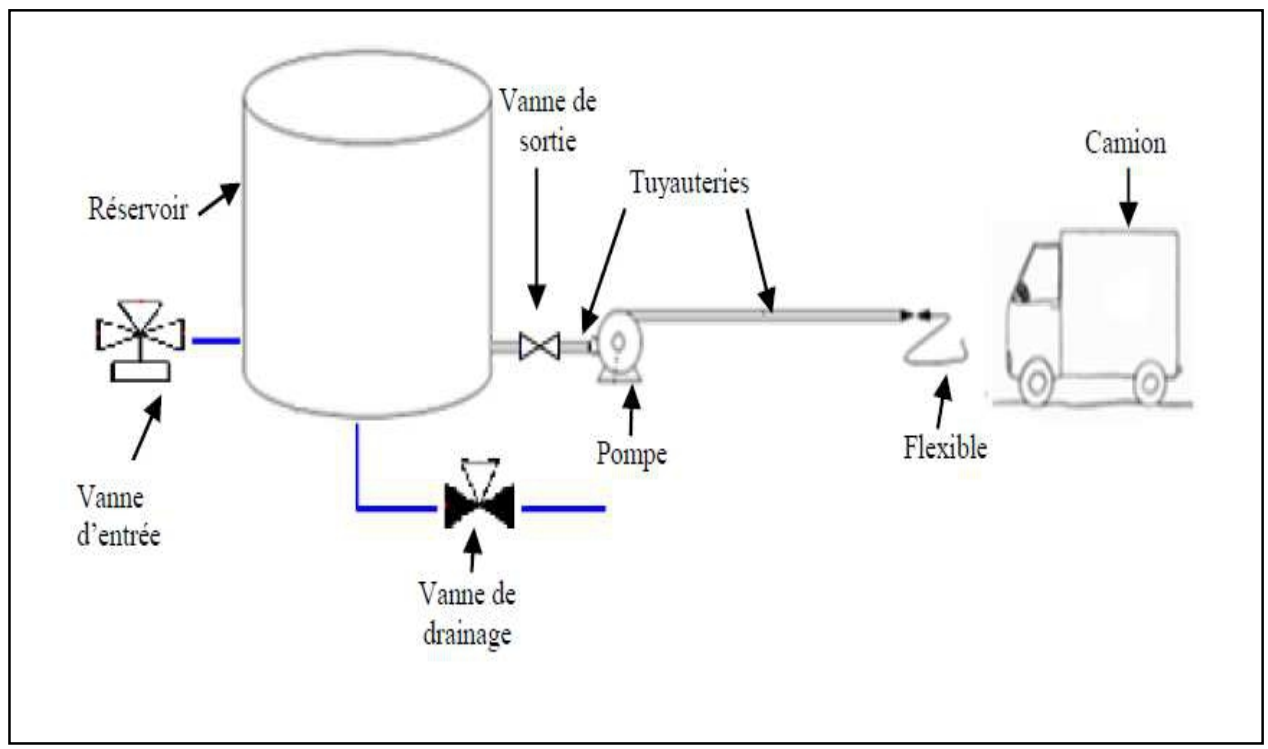

Figure 5 : Schéma de l'installation de déchargement d'ammoniac (Hadni, 2015)

Etape 2 - Sélection d'une unité et identification des objectifs : Comme cité auparavant, nous avons sélectionné le bac de stockage d'ammoniac qui est selon les résultats de l'étude réalisée par Hadni, l'équipement le plus critique pour lequel le risque n'a pas pu être réduit à un niveau acceptable malgré l'optimisation de la périodicité de la maintenance (Hadni, 2015).

A cet effet, l'objectif est de réduire le risque process lié au bac de stockage d'ammoniac à un niveau acceptable. Le bac de stockage d'ammoniac est un réservoir cylindrique en tôle d'acier à toit bombé, calorifugé par une couche de polyuréthane d'épaisseur $120 \mathrm{~mm}$, protégé par un mur de rétention en béton armé d'épaisseur $500 \mathrm{~mm}$. L'espace annulaire entre le bac et le mur de rétention est de $1,5 \mathrm{~m}$.

Etape 3 - Identification initiale des opérations de maintenance : Le scénario du risques process identifié pour le réservoir concerne «la fuite importante et interruptible sur le réservoir d'NH3 (suite à des contraintes de surpression, de corrosion, de surremplissage, etc.) » et ce risque est jugé inacceptable à criticité élevée (Hadni, 2015).

Nous avons défini les tâches de maintenance visant à réduire le risque process «fuite d'ammoniac sur le réservoir » à un niveau acceptable sur la base de l'analyse des causes du scénario de ce risque.

Pour chaque cause identifiée, nous avons défini les opérations de maintenance qui permettront de l'éliminer ou de la maitriser. Ensuite, nous avons identifié les scénarios de risque liés à l'exécution desdites opérations de maintenance. Ces éléments sont définis en concertation avec les 
responsables sécurité et maintenance de la station étudiée et sont présentés dans le tableau 1.

Tableau 1 : Identification initiale des opérations de maintenance et des scénarios de risque associés

\begin{tabular}{|c|c|c|c|}
\hline \multicolumn{2}{|c|}{$\begin{array}{l}\text { RISQUE PROCESS RELATIF AU } \\
\text { RESERVOIR (HADNI, 2015) }\end{array}$} & \multicolumn{2}{|c|}{$\begin{array}{l}\text { IDENTIFICATION INITIALE DES } \\
\text { OPERATIONS DE MAINTENANCE }\end{array}$} \\
\hline Scénario & Causes & $\begin{array}{c}\text { Opérations de } \\
\text { maintenance } \\
\text { identifiées par cause }\end{array}$ & $\begin{array}{c}\text { Scénarios de } \\
\text { Risques }\end{array}$ \\
\hline \multirow{6}{*}{$\begin{array}{l}\text { Fuite } \\
\text { importante et } \\
\text { interruptible } \\
\text { sur le } \\
\text { réservoir } \\
\text { d'NH3 }\end{array}$} & $\begin{array}{l}\text { Défaillance des vannes } \\
\text { (blocage) }\end{array}$ & $\begin{array}{l}\text { Graissage manuel } \\
\text { svstématioue }\end{array}$ & \multirow{6}{*}{$\begin{array}{l}\text { Fuite ou brèche } \\
\text { au niveau du } \\
\text { réservoir } \\
\text { entraînant le } \\
\text { rejet de } \\
\text { l'ammoniac au } \\
\text { moment de } \\
\text { 1'intervention } \\
\text { de l'agent de } \\
\text { Maintenance }\end{array}$} \\
\hline & $\begin{array}{l}\text { Défaillance du système } \\
\text { d'urgence }\end{array}$ & $\begin{array}{l}\text { Vérification et test } \\
\text { local }\end{array}$ & \\
\hline & & Contrôle non destructif & \\
\hline & réservoir & $\begin{array}{l}\text { Traitement } \\
\text { anticorrosion }\end{array}$ & \\
\hline & $\begin{array}{l}\text { Défaillance soupape de } \\
\text { sécurité }\end{array}$ & $\begin{array}{l}\text { Calibration } \\
\text { systématique }\end{array}$ & \\
\hline & $\begin{array}{l}\text { Défaillance instruments de } \\
\text { mesures (débitmètre, } \\
\text { manomètre,...) }\end{array}$ & $\begin{array}{l}\text { Calibration } \\
\text { systématique }\end{array}$ & \\
\hline
\end{tabular}

Etape 4-Evaluation des risques liés aux opérations de maintenance : Nous nous sommes basés sur la matrice de criticité proposée par Texas State University (LBJSC, 2016) pour statuer sur l'acceptabilité des risques liés à l'exécution des opérations de maintenance. Ladite matrice de criticité est présentée ci-dessous (cf. Tableaux $2 \& 3$ ).

Tableau 2 : Matrice de criticité (LBJSC, 2016)

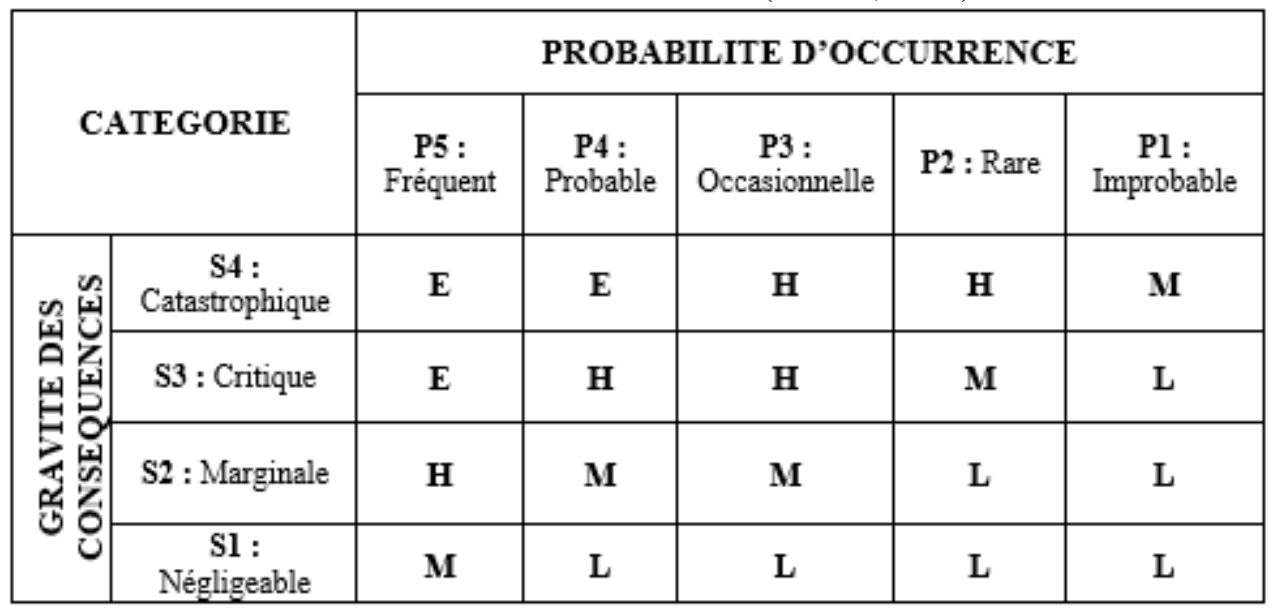


Tableau 3 : Stratégies de management des risques (LBJSC, 2016)

\begin{tabular}{|c|c|c|}
\hline & $\begin{array}{l}\text { DEFINITIONS } \\
\text { DES RISQUES }\end{array}$ & STRATEGIES DE MANAGEMENT DES RISQUES \\
\hline $\mathbf{E}$ & $\begin{array}{l}\text { Risque Extrêmement } \\
\text { Elevé } \\
\text { (Extremely High } \\
\text { Risk) }\end{array}$ & $\begin{array}{l}\text { Dans cette catégorie, les activités présentent des niveaux de } \\
\text { risques inacceptables. Ces risques sont très susceptibles de } \\
\text { se produire. Les organisations devraient éliminer ou } \\
\text { modifier les activités qui ont toujours une note «E» malgré } \\
\text { toutes les actions d'atténuations des risques qui peuvent } \\
\text { s'appliquer. }\end{array}$ \\
\hline $\mathbf{H}$ & $\begin{array}{l}\text { Risque Elevé } \\
\text { (High Risk) }\end{array}$ & $\begin{array}{l}\text { Dans cette catégorie, les activités présentent des } \\
\text { risques potentiellement sérieux susceptibles de se } \\
\text { produire. Il est conseillé d'utiliser des méthodes } \\
\text { proactives de gestion des risques. Les organisations } \\
\text { devraient envisager des moyens de modification ou } \\
\text { d'élimination des risques inacceptables. }\end{array}$ \\
\hline $\mathbf{M}$ & $\begin{array}{l}\text { Risque Modéré } \\
\text { (Moderate Risk) }\end{array}$ & $\begin{array}{l}\text { Dans cette catégorie, les activités présentent des } \\
\text { risques avec une probabilité faible d'occurrence. Les } \\
\text { organisations devraient envisager actions permettant } \\
\text { de gérer ces risques afin d'éviter tout impact négatif. }\end{array}$ \\
\hline $\mathbf{L}$ & $\begin{array}{l}\text { Risque Faible (Low } \\
\text { Risk) }\end{array}$ & $\begin{array}{l}\text { Dans cette catégorie, les activités présentent des } \\
\text { risques faibles et peu probable qu'ils se produisent. } \\
\text { Les organisations peuvent exécuter ces activités } \\
\text { comme planifié. }\end{array}$ \\
\hline
\end{tabular}

L'évaluation des risques est réalisée sur la base d'avis d'expert et en concertation avec le responsable sécurité de la station. Le risque identifié, relatif à l'exécution des opérations de maintenance, correspond à la classe «S4 » (Critique) pour sa conséquence à la classe « P3 » (Occasionnel) pour sa probabilité d'occurrence. L'estimation du risque (Er) est obtenue par le produit des conséquences et des probabilités d'occurrence, à savoir : $\mathbf{E r}=$ Conséquence du risque (S4) x Probabilité d'occurrence (P3).

En se basant sur la matrice de criticité (cf. Tableau 2), le risque lié à l'exécution des opérations de maintenance est élevé $(\mathrm{H})$. Par conséquent, les opérations de maintenance devront être modifiées et/ou améliorées jusqu'à ce que les risques associés auxdites opérations de maintenance soient réduits à un niveau acceptable.

Etape 5 - Modification et amélioration des opérations de maintenance : Les opérations de maintenance améliorées et/ou modifiées ainsi que les nouveaux scénarios de risques concernant l'exécution desdites opérations de maintenance sont définis sur la base d'avis d'expert et en concertation avec le responsable sécurité et maintenance de la station étudié et sont présentés ci- dessous (Cf. Tableau 4). 
Tableau 4 : Les opérations de maintenance modifiées et les scénarios de risque associés

\begin{tabular}{|c|c|c|c|}
\hline $\begin{array}{c}\text { Opérations de } \\
\text { maintenance } \\
\text { initialement } \\
\text { identifiées }\end{array}$ & $\begin{array}{l}\text { Opérations de } \\
\text { maintenance } \\
\text { modifiées }\end{array}$ & $\begin{array}{l}\text { Scénarios de Risques } \\
\text { liés à l'exécution des } \\
\text { opérations de } \\
\text { maintenance } \\
\text { initialement } \\
\text { identifiées }\end{array}$ & $\begin{array}{l}\text { Scénarios de } \\
\text { Risques liés à } \\
\text { l'exécution des } \\
\text { opérations de } \\
\text { maintenance } \\
\text { modifiées }\end{array}$ \\
\hline $\begin{array}{l}\text { Graissage } \\
\text { systématique } \\
\text { manuel }\end{array}$ & $\begin{array}{c}\text { Mise en place } \\
\text { d'une station de } \\
\text { graissage } \\
\text { automatique }\end{array}$ & \multirow{6}{*}{$\begin{array}{c}\text { Fuite ou brèche au } \\
\text { niveau du réservoir } \\
\text { entraînant le rejet de } \\
\text { l'ammoniac au } \\
\text { moment de } \\
\text { l'intervention de } \\
\text { 1'agent de Maintenance }\end{array}$} & $\begin{array}{c}\text { L'agent de } \\
\text { maintenance n'est } \\
\text { plus exposé au risque }\end{array}$ \\
\hline $\begin{array}{l}\text { Vérification et } \\
\text { test local }\end{array}$ & \multirow{5}{*}{$\begin{array}{l}\text { Réalisation des } \\
\text { anciennes } \\
\text { modalités de } \\
\text { maintenance avec } \\
\text { obligatoirement } \\
\text { la formation } \\
\text { sécurité et le port } \\
\text { des équipements } \\
\text { de protection } \\
\text { appropriés par les } \\
\text { agents de } \\
\text { maintenance }\end{array}$} & & \multirow{5}{*}{$\begin{array}{c}\text { Fuite ou brèche au } \\
\text { niveau du réservoir } \\
\text { entraînant le rejet de } \\
\text { 1'ammoniac au } \\
\text { moment de } \\
\text { l'intervention de } \\
\text { l'agent de } \\
\text { Maintenance. Ce } \\
\text { dernier est formé et } \\
\text { porte les équipements } \\
\text { de protection } \\
\text { appropriés }\end{array}$} \\
\hline $\begin{array}{l}\text { Contrôle non } \\
\text { destructive }\end{array}$ & & & \\
\hline $\begin{array}{c}\text { Traitement anti } \\
\text { corrosion }\end{array}$ & & & \\
\hline $\begin{array}{c}\text { Calibration } \\
\text { systématique }\end{array}$ & & & \\
\hline $\begin{array}{c}\text { Calibration } \\
\text { systématique }\end{array}$ & & & \\
\hline
\end{tabular}

Etape 6 - Evaluation de l'acceptabilité des risques liés aux opérations de maintenance modifiées et/ou améliorées : L'évaluation des risques est réalisée sur la base d'avis d'expert et en concertation avec le responsable sécurité de la station. Pour l'opération de maintenance concernant l'utilisation d'une station de graissage automatique, le risque lié à l'exécution de cette opération de maintenance n'est plus présent vu qu'il n'y a plus d'intervention humaine.

Pour les autres opérations de maintenance, les conséquences seront réduites vu que les agents de maintenance seront formés et protégés. La conséquence est réduite de la classe S4 (Critique) à la classe S2 (Marginale).

La mise en place des opérations de maintenance efficaces permettra d'améliorer la fiabilité du réservoir et par conséquent la probabilité d'occurrence du risque de fuite sera réduite. La probabilité d'occurrence sera réduite de la classe P3(Occasionnelle) à la classe P2(Probable).

Sur la base de la matrice de criticité présentée auparavant, le risque lié à l'exécution des nouvelles opérations de maintenance est réduit à un niveau faible (L). Le risque est par conséquent acceptable. 
Etape 7 - Evaluation de l'acceptabilité des coûts liés à la mise en place des opérations de maintenance modifiées et/ou améliorées : L'estimation des coûts liés à la mise en place des opérations de maintenance modifiées et/ou améliorées a été réalisée sur la base de concertation avec une entreprise spécialisée ainsi que sur la base d'avis d'experts (cf. Tableau 5). Le coût est jugé acceptable vu qu'il est largement inférieur par rapport à la conséquence du risque.

Tableau 5 : Estimation du coût des opérations de maintenance modifiées et/ou améliorées

\begin{tabular}{|c|c|c|c|}
\hline Modification apportées & \multicolumn{2}{|c|}{ Estimation du coût } & $\begin{array}{c}\text { Conséquence } \\
\text { du risque } \\
\text { (selon(Hadni, } \\
\text { 2015)) }\end{array}$ \\
\hline $\begin{array}{l}\text { Mise en place d'une station } \\
\text { de graissage automatique }\end{array}$ & \multicolumn{2}{|l|}{ 2.000 Euros } & \multirow{6}{*}{ 100.000 Euros } \\
\hline \multirow{2}{*}{$\begin{array}{l}\text { Conduite de formation en } \\
\text { sécurité au profit des agents } \\
\text { de maintenance }\end{array}$} & $\begin{array}{c}\text { Coût direct : } \\
\text { (Frais de la formation (3 jours)) }\end{array}$ & 3.000 Euros & \\
\hline & $\begin{array}{c}\text { Coût indirect (salaire des } \\
\text { participants, remplacement,...) }\end{array}$ & 2.400 Euros & \\
\hline \multirow{3}{*}{$\begin{array}{l}\text { Equipements de protection } \\
\text { appropriés }\end{array}$} & Masque à gaz (4 Unités) & 240 Euros & \\
\hline & Scaphandre (4 Unités) & 4.800 Euros & \\
\hline & $\begin{array}{c}\text { Appareils Respiratoires isolants - } \\
\text { ARI (4 Unités) }\end{array}$ & 8.800 Euros & \\
\hline TOTAL & \multicolumn{2}{|l|}{ 21.240 Euros } & 100.000 Euros \\
\hline
\end{tabular}

Etape 8 - Vérification de l'atteinte des objectifs : L'objectif défini initialement est de réduire le risque process lié au bac de stockage d'ammoniac à un niveau acceptable. L'évaluation des risques est réalisée sur la base d'avis d'expert. D'une part, après mise en place des opérations de maintenance, les causes identifiés relatives au risque de fuite d'ammoniac au niveau du réservoir seront éliminées et/ou maitrisés et par conséquent la probabilité d'occurrence du risque sera rare (Classe P2 - Rare).

D'autre part, le personnel de l'exploitation doit lui aussi suivre une formation en sécurité et doit être dotés des équipements de protection appropriés. Dans ce cas, les conséquences du risque peuvent être considérées marginales (Classe S2 - Marginale). Dans ce cas, et sur la base de la matrice de criticité présentée auparavant, le risque process sera faible (L) et par conséquent acceptable.

\section{Conclusion}

Cette méthodologie contribue à l'amélioration de la maturité des entreprises en termes de gestion des risques industriels à travers la maitrise des risques liés aux opérations de maintenance tout en prenant en considération les objectifs visés. Toutefois, la disponibilité budgétaire 
représente une contrainte pour la mise en œuvre de cette méthodologie et son efficacité nécessite un retour d'expérience fiable et l'appui d'avis d'expert.

Cependant, la maitrise des risques liés à la maintenance est une condition nécessaire mais pas suffisante pour atteindre un niveau de maturité satisfaisant pour les entreprises en matière de gestion des risques industriels. Pour ceci, il faut entamer en parallèle d'autres actions permettant de maitriser l'ensemble des facteurs clés concernant la maturité en gestion des risques industriels.

\section{References:}

1. AFNOR. (2002). «Maintenance Industrielle». Le fascicule de documentation - FD X 60-000. Association Française de Normalisation (AFNOR). La Plaine Saint-Denis Cedex, France

2. Arunraj, N.S. and Maiti, J. (2007) 'Risk-based maintenanceTechniques and applications', Journal of Hazardous Materials, Vol. 142, pp. 653-661.

3. Barthelemy, B. and Courreges, P. (2012) 'Gestion des risques Méthode d'optimisation globale', 2nd ed., Editions d'organisations, Paris.

4. Elkholti, A.(2015). "Stratégies pour la santé et la sécurité au travail".Séminaire EU-Afrique du Nord, Institut National des conditions de vie au travail, Ministère de l'emploi et des affaires sociales, Maroc.

5. FAT - Fonds des accidents de travail (2010). Les accidents du travail dans le cadre des travaux de Maintenance.

http://www.fat.fgov.be/sites/default/files/assets/FR/Statistiques/

Etudes/Secteurs/Maintenance/notemaintenance2009-fr.pdf .

6. Glade, M. (2005). Modélisation des coûts de cycle de vie : prévision des coûts de maintenance et de la fiabilité Application à l'aéronautique. Thèse de Doctorat. Ecole Centrale de Lyon, France.

7. HADNI, S. (2015) 'Impact de la maintenance sur les risques industriels majeurs', Thèse de Doctorat. Faculté Polytechnique de Mons, Belgique.

8. INRS - Institut National de recherche et de sécurité (2005). " Les accidents liés à la maintenance, étude bibliographique," Note scientifique et Technique $\mathrm{N}^{\circ} 248$. Vandoeuvre Cedex, France.

9. INRS - Institut National de recherche et de sécurité (2013). " Maintenance : des activités à risque," Fiche pratique de sécurité $\mathrm{N}^{\circ} \mathrm{ED}$ 123. Vandoeuvre Cedex, France.

10. INRS - Institut National de recherche et de sécurité (2016). "Dossier : Organisation de la maintenance," Site web: http://www.inrs.fr/risques/maintenance/expositions-risques.html. 
11. ISO - International Organization for Standardization (2009). ISO 31000:2009: Risk management - Principles and guidelines. Geneva, ISO.

12. Jinqiu, H. and Zhang, L. (2014) 'Risk based opportunistic maintenance model for complex mechanical systems', Journal of Expert Systems with Applications, Vol. 41, Issue 6, pp3105-3115.

13. Khan, F. and Haddara, M. (2004) 'Risk-Based Maintenance (RBM): A new approach for process plant inspection and maintenance', Journal of Process Safety Progress Vol.23, N4, pp.252-265.

14. LBJ Student center - Texas state university,. (2016 ) 'Risk Assessment Worksheet' https://www.millikin.edu/sites/default/files/documents/risk_assessme nt_matrix.pdf (Accessed 11 July 2016).

15. Madika ,C. (2011). "Rapport d'Evaluation et analyse du système national de couverture sociale contre les accidents du travail et les maladies professionnelles au Maroc,". Accord de coopération GIPSPSI 2010- 2012, Ministère de l'Emploi et de la Formation Professionnelle, Maroc.

16. Monchy, F. and Vernier, J.P. (2010) 'Maintenance - Méthodes et organisations', 3rd ed., Dunod, Paris, pp. 451-509.

17. Nguyen, T. P. K. (2012) 'Politiques de maintenance et d'investissement sous évolution technologique incertaine', Thèse de Doctorat. Ecole Centrale de Nantes, France.

18. Nilsson, F. (2003) 'Risk-based approach to plant life management', Journal of Nuclear Engineering and Design, Vol.221, Issues 1-3, pp.293-300.

19. Nissoul, H., Riane, F. and Mousrij, A. (2017) 'Proposition d'un modèle de maturité pour la gestion des risques: application au secteur industriel au Maroc', Revue assurance et gestion des risques. Vol. 84 (1-2) 2017. Université LAVAL. Canada.

20. OIT - Organisation Internationale du Travail. (2013). Faits et chiffres sur la santé et la sécurité au travail. Fiche d'Information. Retrieved from http://www.ilo.org/global/about-the-ilo/newsroom/mediacentre/issue-briefs/WCMS_206596/lang--fr/index.htm.

21. OIT - Organisation Internationale du Travail. (2015). Enquêtes sur les accidents du travail et les maladies professionnelles: Guide pratique à l'intention des inspecteurs du travail. ISBN: 978-92-2229419-0 (print), ISBN: 978-92-2-229420-6 (web pdf). Genève.

22. OIT - Organisation Internationale du Travail. (2017). Sécurité et santé au travail. http://www.ilo.org/global/topics/safety-and-health-at-work/lang-fr/index.htm 
23. Ray, P.S., Batson, R.G., Weems, W.H., Wan, Q., Sorock, G.S., Matz, S. and Cotnam, J. (2000) 'Impact of maintenance function on plant safety', Journal of professional safety - American society of safety Engineers, August, pp. 45-48.

24. Sadiq, R.., Khan, F. and Haddara, M. (2004) 'Risk-based inspection and maintenance (RBIM) Multi-attribute Decision-making with Aggregative Risk Analysis', Journal of Process Safety and Environmental Protection, Vol. 82, Issue 6, pp. 398-411.

25. Vaurio, J.K. (1995) 'Optimization of test and maintenance intervals based on risk and cost', Journal of Reliability Engineering and System Safety, Vol. 49, Issue 1, pp. 23-36. 